\title{
Effect of Sodium Azide in Yield and Yield Contributing Traits of Confectionary Sunflower (Helianthus annuus L.)
}

\author{
K. Sai Phanindra*, V.N. Toprope and N.R. Thakur \\ Department of Genetics and Plant Breeding, College of Agriculture, Latur, VNMKV, \\ Parbhani, Maharashtra-413512, India \\ *Corresponding author
}

\section{A B S T R A C T}

Keywords

Sunflower

(Helianthus annuus

L.), Sodium Azide,

Genotypes

Article Info

Accepted:

16 August 2018

Available Online:

10 September 2018
Confectionary sunflower genotypes, EC 625693, EC 318761 and SCG 62 were treated with sodium azide at $100 \mathrm{ppm}, 200 \mathrm{ppm}$ and $300 \mathrm{ppm}$, respectively. In $\mathrm{M}_{2}$ generation, observations were recorded on eight quantitative characters viz., days to $50 \%$ flowering, days to maturity, plant height, head diameter, seed filling percentage, hull content, 100 grain weight and seed yield per plant. The treatment $100 \mathrm{ppm}$ had high mean value for yield and yield contributing characters like head diameter, seed filling percentage, 100 grain weight and seed yield per plant. However, the SCG 62 mutant populations showed negative response for most of the characters. The estimates of GCV, PCV, heritability and genetic advance as percent of mean among treatments were maximum in treatments 300 ppm for head diameter, seed filling percentage and seed yield per plant. Whereas $100 \mathrm{ppm}$ for 100 grain weight and 200 ppm for seed yield per plant also exhibited high estimates of GCV, PCV, heritability and genetic advance as percent of mean in all three mutant populations and selection for these characters enhances genetic improvement.

\section{Introduction}

Sunflower (Helianthus annuus L.) is one of the widely grown oilseed crop around the world. It is one of the useful contingent crops especially under rainfed cultivation owing to its day neutral nature and tolerance to temperature and soil moisture regimes (Natikar et al., 2013). Soybean, groundnut, rapeseed, mustard are the major competitors of Sunflower at global level. India is the fourth largest sunflower growing country among the world. Sunflower oil contains 40$45 \%$ of edible along with high concentration of poly unsaturated fatty acids and it is recommended for heart patients. Two types of Sunflower are available in market viz., oil seed and confectionary type. Confectionary type of Sunflower is largely grown in Argentina. In European countries, kernels of confectionary sunflower are used in bakery products. As the seeds of confectionary sunflower is larger compared to oil seed type, the main aim of confectionary sunflower breeding is to develop low hull content lines with high yielding ability. The success of any crop improvement programme in meeting the various objectives is dependent upon availability of necessary genetic variation and to produce with latest technologies present in 
global era. Coefficient of variability, heritability and genetic advance helps to find the amount of genetic variability in population. Sodium azide is safe and it creates point mutations in the genome of plants through metabolite and thus produced protein in mutant plants has different function compared to the normal plants (Khan and Goyal, 2009).

\section{Materials and Methods}

For current study, the seeds of three Confectionary sunflower genotypes viz., EC 625693, EC 318761, SCG 62 were treated with different concentrations (100 ppm, 200 ppm and $300 \mathrm{ppm}$ ) of sodium azide for four hours in distilled water along with control. The treated seeds were sown for raising $\mathrm{M}_{1}$ generation during Rabi 2016 at Experimental farm, College of Agriculture, Latur. The seeds of selected $\mathrm{M}_{1}$ plants were sown in randomized block design in two replications with control. The plot was sown on the basis of plant to row progenies with a spacing of 60 $\times 30 \mathrm{~cm}$. The field conditions were similar as that of $M_{1}$ generation. All recommended package of practices was followed. The observations were recorded on eight quantitative characters viz., days to $50 \%$ flowering, days to maturity, plant height, head diameter, seed filling percentage, hull content, 100 grain weight and seed yield per plant. The analysis was based on the model suggested by Panse and Sukhatme (1985). The genotypic (GCV) and phenotypic (PCV) coefficients of variation (Burton and Devane, 1953), heritability in broad sense (Johnson et al., 1955) and genetic advance (Allard-1960) were computed by using standard statistical methods.

\section{Results and Discussion}

Analysis of variance showed significant difference among mutant populations compared with control and it creates an ample scope for selecting promising mutant lines with agronomic attributes. The data on range, mean, shift in mean, genotypic coefficient of variation, phenotypic coefficient of variation, heritability, genetic advance and genetic advance as percent of mean for yield and yield contributing characters among treatments were presented in Table 1. A wide range of variability was obtained in mutant populations. The means values were varied in both positive and negative direction from control in $\mathrm{M}_{2}$ generation. Induced mutagenesis (Table 1) leads negative shift in mean values for days to $50 \%$ flowering and days to maturity in all three genotypes with all treatments of sodium azide. Similar observations have been also observed in SCG 62 for head diameter, seed filling percentage and seed yield per plant and EC 318761 for hull content. The results are in accordance with earlier findings of Cvejic et al., (2015) in sunflower and Gunasekaran and Pavadai (2015) in groundnut for days to $50 \%$ flowering and Khan and Goyal (2009) in mungbean and Kulmi and Mogali (2016) in linseed for days to maturity. Head diameter and test weight of EC 625693 in all three treatments showed positive shift in mean values as compared to their control. The results are in conformity with findings of Jagadeesan et al., (2008) and Cvejic et al., (2015) for head diameter and Jagadeesan et al., (2008) for test weight. In both the genotypes, EC 625693 and EC 318761100 ppm treatment of sodium azide increased mean values for head diameter, seed filling percentage, test weight and seed yield per plant. However, the remaining treatments showed either positive or negative shift in mean values as compared to their respective control for most of the traits. Cvejic et al., (2015) in sunflower and Roychowdhury et al., (2012) in mungbean reported positive shift in mean value for seed yield per plant in mutant population. 
Table.1 Estimates of range, mean, shift in mean, phenotypic and genotypic coefficient of variation, heritability and genetic advance among mutant plants for yield and yield contributing characters in $\mathrm{M}_{2}$ generation of confectionary sunflower

\begin{tabular}{|c|c|c|c|c|c|c|c|c|c|c|c|}
\hline $\begin{array}{l}\text { Sr. } \\
\text { No. }\end{array}$ & Parameters & $\begin{array}{l}\text { Genotype } \\
\text { name }\end{array}$ & $\begin{array}{c}\text { Concentration/ } \\
\text { dose }\end{array}$ & Range & Mean \pm S.E & $\begin{array}{c}\text { Shift in } \\
\text { mean }\end{array}$ & $\begin{array}{l}\text { GCV } \\
(\%)\end{array}$ & $\begin{array}{l}\text { PCV } \\
(\%)\end{array}$ & $\begin{array}{l}\text { Heritability } \\
\text { (B.S) }(\%)\end{array}$ & $\begin{array}{l}\text { Genetic } \\
\text { advance }\end{array}$ & $\begin{array}{c}\text { Genetic advance } \\
\text { as } \% \text { of mean }\end{array}$ \\
\hline \multirow{12}{*}{1.} & \multirow{11}{*}{$\begin{array}{c}\text { Days to } \\
50 \% \\
\text { flowering }\end{array}$} & \multirow{3}{*}{$\begin{array}{c}\text { EC } \\
625693\end{array}$} & Control & $66.50-72.00$ & $68.37 \pm 0.87$ & & 1.88 & 2.70 & 51.83 & 1.97 & 2.89 \\
\hline & & & $100 \mathrm{ppm}$ & $54.50-70.50$ & $62.10 \pm 2.58$ & -6.27 & 5.38 & 8.12 & 43.88 & 4.56 & 7.34 \\
\hline & & & 300 ppm & $62.00-72.50$ & $65.17 \pm 1.50$ & -3.2 & 2.85 & 4.38 & 42.31 & 2.49 & 3.82 \\
\hline & & \multirow{4}{*}{$\begin{array}{c}\text { EC } \\
318761\end{array}$} & Control & $60.50-67.00$ & $63.96 \pm 0.92$ & & 2.16 & 3.03 & 50.65 & 2.02 & 3.17 \\
\hline & & & 100 ppm & $57.50-67.00$ & $62.11 \pm 1.63$ & -1.85 & 4.41 & 5.91 & 55.67 & 4.21 & 6.78 \\
\hline & & & 200 ppm & $57.50-66.00$ & $63.27 \pm 1.24$ & -0.69 & 3.30 & 4.41 & 56.14 & 3.22 & 5.09 \\
\hline & & & $300 \mathrm{ppm}$ & $59.00-64.00$ & $62.47 \pm 0.83$ & -1.49 & 2.17 & 2.91 & 55.60 & 2.08 & 3.34 \\
\hline & & \multirow[t]{4}{*}{ SCG 62} & Control & $61.50-65.00$ & $63.79 \pm 0.43$ & & 1.42 & 1.73 & 66.87 & 1.52 & 2.39 \\
\hline & & & 100 ppm & $60.00-65.00$ & $62.94 \pm 0.62$ & -0.85 & 2.33 & 2.76 & 71.10 & 2.55 & 4.05 \\
\hline & & & 200 ppm & $61.00-65.50$ & $62.75 \pm 0.67$ & -1.04 & 2.91 & 3.35 & 75.47 & 3.27 & 5.21 \\
\hline & & & 300 ppm & $61.00-66.00$ & $63.41 \pm 0.67$ & -0.37 & 2.54 & 3.03 & 70.59 & 2.79 & 4.40 \\
\hline & \multicolumn{2}{|c|}{ C.D. $(p=0.05)$} & & & & 1.45 & & & & & \\
\hline \multirow{12}{*}{2.} & \multirow{11}{*}{$\begin{array}{l}\text { Days to } \\
\text { maturity }\end{array}$} & \multirow{3}{*}{$\begin{array}{c}\text { EC } \\
625693\end{array}$} & Control & $89.00-93.50$ & $91.58 \pm 0.62$ & & 1.05 & 1.46 & 52.34 & 1.44 & 1.57 \\
\hline & & & $100 \mathrm{ppm}$ & $86.00-90.50$ & $88.63 \pm 0.83$ & -2.95 & 1.29 & 1.88 & 47.10 & 1.62 & 1.83 \\
\hline & & & $300 \mathrm{ppm}$ & $82.00-93.50$ & $91.06 \pm 1.35$ & -0.52 & 1.94 & 2.88 & 45.06 & 2.44 & 2.68 \\
\hline & & \multirow{4}{*}{$\begin{array}{c}\text { EC } \\
318761\end{array}$} & Control & $92.50-97.00$ & $94.17 \pm 0.76$ & & 1.14 & 1.65 & 47.96 & 1.53 & 1.63 \\
\hline & & & 100 ppm & $91.00-94.00$ & $91.94 \pm 0.28$ & -2.23 & 0.86 & 0.98 & 77.59 & 1.43 & 1.56 \\
\hline & & & 200 ppm & $88.50-94.50$ & $91.68 \pm 0.90$ & -2.49 & 2.00 & 2.48 & 65.43 & 3.06 & 3.34 \\
\hline & & & 300 ppm & $91.00-94.50$ & $93.05 \pm 0.64$ & -1.11 & 0.87 & 1.33 & 42.89 & 1.09 & 1.17 \\
\hline & & \multirow[t]{4}{*}{ SCG 62} & Control & $92.00-95.00$ & $93.75 \pm 0.62$ & & 0.99 & 1.40 & 50.66 & 1.37 & 1.46 \\
\hline & & & 100 ppm & $92.00-95.50$ & $92.61 \pm 0.55$ & -1.14 & 1.12 & 1.43 & 61.42 & 1.68 & 1.81 \\
\hline & & & $200 \mathrm{ppm}$ & $93.00-94.50$ & $93.25 \pm 0.19$ & -0.5 & 0.62 & 0.69 & 80.00 & 1.06 & 1.14 \\
\hline & & & 300 ppm & $90.00-96.00$ & $93.50 \pm 0.69$ & -0.25 & 2.28 & 2.55 & 80.00 & 3.92 & 4.19 \\
\hline & \multicolumn{2}{|c|}{ C.D. $(p=0.05)$} & & & & 0.83 & & & & & \\
\hline
\end{tabular}




\begin{tabular}{|c|c|c|c|c|c|c|c|c|c|c|c|}
\hline \multicolumn{12}{|c|}{ Continued... } \\
\hline $\begin{array}{l}\text { Sr. } \\
\text { No. }\end{array}$ & Parameters & $\begin{array}{l}\text { Genotype } \\
\text { name }\end{array}$ & $\begin{array}{c}\text { Concentration/ } \\
\text { dose }\end{array}$ & Range & Mean \pm S.E & $\begin{array}{l}\text { Shift } \\
\text { in } \\
\text { mean }\end{array}$ & $\begin{array}{r}\text { GCV } \\
(\%)\end{array}$ & $\begin{array}{r}\text { PCV } \\
(\%)\end{array}$ & $\begin{array}{l}\text { Heritability } \\
\text { (B.S) }(\%)\end{array}$ & $\begin{array}{l}\text { Genetic } \\
\text { advance }\end{array}$ & $\begin{array}{c}\text { Genetic } \\
\text { advance } \\
\text { as } \% \text { of } \\
\text { mean }\end{array}$ \\
\hline \multirow{12}{*}{3.} & \multirow{12}{*}{$\begin{array}{l}\text { Plant height } \\
(\mathrm{cm})\end{array}$} & \multirow{4}{*}{$\begin{array}{c}\mathrm{EC} \\
625693\end{array}$} & Control & $128.5-165.0$ & $150.94 \pm 6.54$ & & 6.36 & 9.02 & 49.74 & 13.96 & 9.25 \\
\hline & & & 100 ppm & $107.5-191.0$ & $146.18 \pm 11.42$ & -4.76 & 11.16 & 15.98 & 48.77 & 23.47 & 16.06 \\
\hline & & & 200 ppm & $125.5-196.0$ & $149.58 \pm 13.28$ & -1.36 & 12.68 & 18.24 & 48.30 & 27.15 & 18.15 \\
\hline & & & 300 ppm & $127.0-210.0$ & $162.82 \pm 15.82$ & +11.89 & 10.41 & 17.49 & 35.43 & 20.78 & 12.76 \\
\hline & & \multirow{4}{*}{$\begin{array}{c}\mathrm{EC} \\
318761\end{array}$} & Control & $154.5-197.0$ & $180.04 \pm 7.73$ & & 7.45 & 9.79 & 57.97 & 21.04 & 11.68 \\
\hline & & & 100 ppm & $142.5-178.0$ & $167.67 \pm 4.06$ & -12.37 & 5.70 & 6.76 & 71.04 & 16.58 & 9.89 \\
\hline & & & 200 ppm & $158.5-189.5$ & $181.31 \pm 2.27$ & +1.28 & 4.18 & 4.57 & 83.54 & 14.27 & 7.87 \\
\hline & & & 300 ppm & $142.5-202.5$ & $180.41 \pm 10.38$ & +0.37 & 6.94 & 10.89 & 40.66 & 16.46 & 9.12 \\
\hline & & \multirow{4}{*}{ SCG 62} & Control & $157.5-166.5$ & $161.17 \pm 1.74$ & & 1.54 & 2.21 & 48.18 & 3.54 & 2.20 \\
\hline & & & 100 ppm & $129.5-180.0$ & $147.44 \pm 5.43$ & -13.73 & 9.51 & 11.00 & 74.76 & 24.98 & 16.94 \\
\hline & & & 200 ppm & $138.5-176.0$ & $156.08 \pm 5.40$ & -5.09 & 8.50 & 10.05 & 71.60 & 23.14 & 14.82 \\
\hline & & & 300 ppm & $120.0-195.5$ & $166.33 \pm 10.69$ & +5.16 & 14.18 & 17.33 & 66.97 & 39.77 & 23.91 \\
\hline & \multicolumn{2}{|c|}{ C.D. $(p=0.05)$} & & & & 9.05 & & & & & \\
\hline \multirow{13}{*}{4.} & \multirow{12}{*}{$\begin{array}{c}\text { Head } \\
\text { diameter } \\
(\mathrm{cm})\end{array}$} & \multirow{4}{*}{$\begin{array}{c}\mathrm{EC} \\
625693\end{array}$} & Control & $9.85-19.25$ & $15.72 \pm 0.94$ & & 16.03 & 18.33 & 76.55 & 4.54 & 28.90 \\
\hline & & & 100 ppm & $13.50-25.25$ & $18.88 \pm 2.20$ & +3.16 & 14.98 & 22.74 & 43.38 & 3.84 & 20.32 \\
\hline & & & 200 ppm & $13.50-22.00$ & $16.79 \pm 1.54$ & +1.07 & 13.79 & 19.38 & 50.66 & 3.39 & 20.22 \\
\hline & & & 300 ppm & $11.50-24.50$ & $16.54 \pm 2.40$ & +0.82 & 15.79 & 26.27 & 36.16 & 3.24 & 19.57 \\
\hline & & \multirow{4}{*}{$\begin{array}{c}\mathrm{EC} \\
318761\end{array}$} & Control & $14.00-18.50$ & $16.56 \pm 0.79$ & & 8.13 & 10.73 & 57.32 & 2.10 & 12.67 \\
\hline & & & 100 ppm & $16.60-20.35$ & $18.66 \pm 0.62$ & +2.11 & 5.53 & 7.43 & 55.34 & 1.58 & 8.47 \\
\hline & & & 200 ppm & $14.70-16.30$ & $15.57 \pm 0.28$ & -0.99 & 2.72 & 3.82 & 50.80 & 0.62 & 3.99 \\
\hline & & & 300 ppm & $10.45-17.55$ & $14.36 \pm 1.22$ & -2.2 & 10.13 & 15.98 & 40.23 & 1.90 & 13.24 \\
\hline & & \multirow{4}{*}{ SCG 62} & Control & $14.95-18.25$ & $16.60 \pm 0.51$ & & 4.84 & 6.62 & 53.39 & 1.21 & 7.29 \\
\hline & & & 100 ppm & $10.70-19.00$ & $16.36 \pm 1.40$ & -0.24 & 14.93 & 19.68 & 57.58 & 3.82 & 23.34 \\
\hline & & & $200 \mathrm{ppm}$ & $13.20-18.90$ & $15.80 \pm 0.79$ & -0.81 & 11.86 & 14.17 & 70.01 & 3.23 & 20.44 \\
\hline & & & 300 ppm & $7.70-18.55$ & $13.92 \pm 1.49$ & -2.69 & 23.73 & 28.97 & 67.11 & 5.58 & 40.05 \\
\hline & \multicolumn{2}{|c|}{ C.D. $(p=0.05)$} & & & & 1.23 & & & & & \\
\hline
\end{tabular}




\begin{tabular}{|c|c|c|c|c|c|c|c|c|c|c|c|}
\hline \multirow[b]{2}{*}{$\begin{array}{l}\text { Sr. } \\
\text { No. }\end{array}$} & \multirow[b]{2}{*}{ Parameters } & \multirow[b]{2}{*}{$\begin{array}{l}\text { Genotype } \\
\text { name }\end{array}$} & \multirow[b]{2}{*}{$\begin{array}{c}\text { Concentration/ } \\
\text { dose }\end{array}$} & \multirow[b]{2}{*}{ Range } & \multirow[b]{2}{*}{ Mean \pm S.E } & \multirow[b]{2}{*}{$\begin{array}{c}\text { Shift } \\
\text { in } \\
\text { mean }\end{array}$} & \multirow[b]{2}{*}{$\begin{array}{l}\text { GCV } \\
(\%)\end{array}$} & \multirow[b]{2}{*}{$\begin{array}{l}\text { PCV } \\
(\%)\end{array}$} & \multirow[b]{2}{*}{$\begin{array}{l}\text { Heritability } \\
\text { (B.S) }(\%)\end{array}$} & \multirow[b]{2}{*}{$\begin{array}{l}\text { Continu } \\
\text { Genetic } \\
\text { advance }\end{array}$} & \multirow[b]{2}{*}{$\begin{array}{l}\text { d... } \\
\text { Genetic } \\
\text { advance } \\
\text { as \% of } \\
\text { mean }\end{array}$} \\
\hline & & & & & & & & & & & \\
\hline \multirow{12}{*}{5.} & \multirow{12}{*}{$\begin{array}{l}\text { Seed filling } \\
(\%)\end{array}$} & \multirow{4}{*}{$\begin{array}{c}\mathrm{EC} \\
625693\end{array}$} & Control & $47.15-81.70$ & $60.24 \pm 5.52$ & & 13.00 & 18.78 & 47.95 & 11.17 & 18.55 \\
\hline & & & 100 ppm & $30.88-89.91$ & $66.86 \pm 3.61$ & +6.62 & 22.32 & 23.68 & 88.81 & 28.91 & 43.32 \\
\hline & & & 200 ppm & $43.39-84.06$ & $60.88 \pm 5.35$ & +0.64 & 16.45 & 20.96 & 61.62 & 16.20 & 26.61 \\
\hline & & & 300 ppm & $18.74-71.51$ & $46.05 \pm 9.57$ & -14.19 & 25.91 & 39.68 & 42.65 & 16.05 & 34.86 \\
\hline & & \multirow{4}{*}{$\begin{array}{c}\text { EC } \\
318761\end{array}$} & Control & $61.35-75.35$ & $68.85 \pm 3.61$ & & 13.37 & 19.15 & 48.74 & 7.47 & 19.30 \\
\hline & & & 100 ppm & $29.78-59.27$ & $40.36 \pm 2.76$ & -28.49 & 22.28 & 24.53 & 82.51 & 16.83 & 41.69 \\
\hline & & & 200 ppm & $25.82-52.74$ & $38.74 \pm 3.80$ & -30.11 & 19.83 & 24.60 & 65.03 & 12.77 & 32.95 \\
\hline & & & 300 ppm & $23.58-45.04$ & $34.56 \pm 3.85$ & -34.29 & 15.53 & 22.46 & 47.80 & 7.64 & 22.11 \\
\hline & & \multirow[t]{4}{*}{ SCG 62} & Control & $55.60-95.47$ & $80.42 \pm 6.16$ & & 16.08 & 19.67 & 66.85 & 21.78 & 27.09 \\
\hline & & & 100 ppm & $44.02-80.89$ & $66.06 \pm 4.43$ & -14.35 & 16.87 & 19.65 & 73.75 & 19.72 & 29.85 \\
\hline & & & 200 ppm & $47.07-82.58$ & $67.58 \pm 3.48$ & -12.83 & 17.09 & 18.87 & 82.08 & 21.56 & 31.90 \\
\hline & & & 300 ppm & $47.86-94.10$ & $69.07 \pm 5.95$ & -11.35 & 19.99 & 24.03 & 69.17 & 23.66 & 34.25 \\
\hline & \multicolumn{2}{|c|}{ C.D. $(p=0.05)$} & & & & 5.61 & & & & & \\
\hline \multirow{13}{*}{6.} & \multirow{12}{*}{$\begin{array}{l}\text { Hull content } \\
\qquad(\%)\end{array}$} & \multirow{4}{*}{$\begin{array}{c}\mathrm{EC} \\
625693\end{array}$} & Control & $36.25-50.30$ & $43.32 \pm 2.36$ & & 8.70 & 11.86 & 53.80 & 5.69 & 13.15 \\
\hline & & & 100 ppm & $35.00-50.00$ & $44.10 \pm 2.74$ & +0.78 & 7.86 & 12.02 & 42.71 & 4.67 & 10.58 \\
\hline & & & 200 ppm & $37.50-52.50$ & $45.00 \pm 3.02$ & +1.68 & 9.77 & 13.91 & 49.33 & 6.36 & 14.14 \\
\hline & & & 300 ppm & $31.50-47.00$ & $40.15 \pm 1.83$ & -3.17 & 6.50 & 9.25 & 49.43 & 3.78 & 9.42 \\
\hline & & \multirow{4}{*}{$\begin{array}{c}\text { EC } \\
318761\end{array}$} & Control & $42.60-46.50$ & $44.58 \pm 0.73$ & & 2.95 & 3.81 & 59.69 & 2.09 & 4.69 \\
\hline & & & 100 ppm & $40.50-44.00$ & $42.44 \pm 0.61$ & -2.14 & 2.40 & 3.24 & 55.15 & 1.56 & 3.68 \\
\hline & & & 200 ppm & $40.00-44.50$ & $42.09 \pm 0.65$ & -2.49 & 3.07 & 3.84 & 64.11 & 2.13 & 5.07 \\
\hline & & & 300 ppm & $41.00-45.50$ & $42.65 \pm 0.73$ & -1.93 & 2.10 & 3.26 & 41.11 & 1.19 & 2.78 \\
\hline & & \multirow[t]{4}{*}{ SCG 62} & Control & $39.50-43.00$ & $41.00 \pm 0.66$ & & 2.30 & 3.31 & 48.15 & 1.34 & 3.28 \\
\hline & & & 100 ppm & $37.00-43.00$ & $39.22 \pm 0.87$ & -1.78 & 4.10 & 5.29 & 60.00 & 2.56 & 6.54 \\
\hline & & & 200 ppm & $38.50-42.50$ & $39.91 \pm 0.53$ & -1.08 & 3.71 & 4.25 & 76.30 & 2.67 & 6.69 \\
\hline & & & 300 ppm & $40.50-44.00$ & $42.25 \pm 0.53$ & +2.15 & 3.29 & 3.83 & 73.89 & 2.46 & 5.83 \\
\hline & \multicolumn{2}{|c|}{ C.D. $(p=0.05)$} & & & & 1.15 & & & & & \\
\hline
\end{tabular}


Continued...

\begin{tabular}{|c|c|c|c|c|c|c|c|c|c|c|c|}
\hline $\begin{array}{l}\text { Sr. } \\
\text { No. }\end{array}$ & Parameters & $\begin{array}{l}\text { Genotype } \\
\text { name }\end{array}$ & $\begin{array}{c}\text { Concentration/ } \\
\text { dose }\end{array}$ & Range & Mean \pm S.E & $\begin{array}{l}\text { Shift } \\
\text { in } \\
\text { mean }\end{array}$ & $\begin{array}{l}\text { GCV } \\
(\%)\end{array}$ & $\begin{array}{l}\text { PCV } \\
(\%)\end{array}$ & $\begin{array}{c}\text { Heritability } \\
\text { (B.S) }(\%)\end{array}$ & $\begin{array}{l}\text { Genetic } \\
\text { advance }\end{array}$ & $\begin{array}{c}\text { Genetic } \\
\text { advance } \\
\text { as \% of } \\
\text { mean }\end{array}$ \\
\hline \multirow{12}{*}{7.} & \multirow{12}{*}{$\begin{array}{l}100 \\
\text { grain weight } \\
(\mathrm{gm})\end{array}$} & \multirow{4}{*}{$\begin{array}{c}\mathrm{EC} \\
625693\end{array}$} & Control & $6.65-9.10$ & $7.82 \pm 0.36$ & & 8.58 & 11.00 & 60.76 & 1.07 & 13.77 \\
\hline & & & 100 ppm & $6.75-9.50$ & $8.08 \pm 0.49$ & +0.26 & 7.63 & 11.65 & 42.91 & 0.83 & 10.30 \\
\hline & & & 200 ppm & $6.50-9.35$ & $8.18 \pm 0.39$ & +0.36 & 7.22 & 10.14 & 50.76 & 0.87 & 10.60 \\
\hline & & & 300 ppm & $7.70-11.65$ & $9.55 \pm 0.61$ & +1.73 & 9.34 & 13.19 & 50.14 & 1.30 & 13.62 \\
\hline & & \multirow{4}{*}{$\begin{array}{c}\mathrm{EC} \\
318761\end{array}$} & Control & $8.15-10.25$ & $9.66 \pm 0.31$ & & 5.36 & 7.17 & 55.89 & 0.80 & 8.26 \\
\hline & & & 100 ppm & $8.50-10.90$ & $9.75 \pm 0.39$ & +0.09 & 7.47 & 9.64 & 60.07 & 1.16 & 11.93 \\
\hline & & & 200 ppm & $7.65-9.45$ & $8.80 \pm 0.27$ & -0.86 & 4.73 & 6.55 & 52.08 & 0.62 & 7.03 \\
\hline & & & 300 ppm & $8.70-11.40$ & $9.61 \pm 0.32$ & -0.05 & 6.52 & 8.16 & 63.84 & 1.03 & 10.73 \\
\hline & & \multirow[t]{4}{*}{ SCG 62} & Control & $5.85-6.85$ & $6.27 \pm 0.17$ & & 4.00 & 5.63 & 50.75 & 0.36 & 5.88 \\
\hline & & & 100 ppm & $5.90-6.91$ & $6.44 \pm 0.15$ & +0.17 & 4.47 & 5.65 & 62.67 & 0.47 & 7.29 \\
\hline & & & 200 ppm & $5.80-6.57$ & $6.32 \pm 0.10$ & +0.06 & 4.05 & 4.77 & 72.14 & 0.45 & 7.09 \\
\hline & & & 300 ppm & $5.86-6.42$ & $6.16 \pm 0.09$ & -0.11 & 3.52 & 4.18 & 71.06 & 0.38 & 6.11 \\
\hline & \multicolumn{2}{|c|}{ C.D. $(p=0.05)$} & & & & 0.53 & & & & & \\
\hline \multirow{12}{*}{8.} & \multirow{12}{*}{$\begin{array}{l}\text { Seed yield } \\
\text { per plant } \\
\text { (gm) }\end{array}$} & \multirow{4}{*}{$\begin{array}{c}\mathrm{EC} \\
625693\end{array}$} & Control & $22.90-30.80$ & $26.24 \pm 1.34$ & & 7.38 & 10.58 & 48.72 & 2.78 & 10.61 \\
\hline & & & 100 ppm & $17.74-33.98$ & $27.79 \pm 2.28$ & +1.55 & 12.29 & 17.20 & 51.05 & 5.03 & 18.09 \\
\hline & & & 200 ppm & $14.24-36.15$ & $23.30 \pm 3.10$ & -2.94 & 19.07 & 27.40 & 48.46 & 6.37 & 27.35 \\
\hline & & & 300 ppm & $12.11-36.82$ & $22.82 \pm 3.93$ & -3.42 & 20.64 & 32.37 & 40.68 & 6.19 & 27.12 \\
\hline & & \multirow{4}{*}{$\begin{array}{c}\mathrm{EC} \\
318761\end{array}$} & Control & $20.70-28.15$ & $24.83 \pm 1.18$ & & 7.03 & 9.92 & 50.16 & 2.55 & 10.25 \\
\hline & & & 100 ppm & $21.05-31.40$ & $25.34 \pm 1.43$ & +0.5 & 9.54 & 12.74 & 56.10 & 3.73 & 14.72 \\
\hline & & & 200 ppm & $12.50-27.15$ & $19.25 \pm 2.05$ & -5.59 & 17.10 & 23.30 & 53.84 & 4.97 & 25.84 \\
\hline & & & 300 ppm & $12.27-18.75$ & $16.30 \pm 1.11$ & -8.54 & 8.82 & 13.27 & 44.24 & 1.97 & 12.09 \\
\hline & & \multirow[t]{4}{*}{ SCG 62} & Control & $18.80-33.65$ & $26.92 \pm 2.11$ & & 13.96 & 18.15 & 59.12 & 5.95 & 22.11 \\
\hline & & & 100 ppm & $16.40-36.20$ & $25.10 \pm 3.30$ & -1.82 & 22.29 & 29.77 & 56.06 & 8.63 & 34.38 \\
\hline & & & 200 ppm & $12.55-28.05$ & $22.11 \pm 2.04$ & -4.81 & 22.15 & 26.39 & 70.43 & 8.47 & 38.29 \\
\hline & & & 300 ppm & $12.80-24.55$ & $21.47 \pm 1.53$ & -5.45 & 19.22 & 22.18 & 75.09 & 7.37 & 34.31 \\
\hline & \multicolumn{2}{|c|}{ C.D. $(p=0.05)$} & & & & 2.18 & & & & & \\
\hline
\end{tabular}


It is evident from the results for days to $50 \%$ flowering and days to maturity in treated populations, had low genotypic and phenotypic coefficient of variation which reveals the presence of narrow range variability for these characters and restricting the scope of selection. Similar results in sunflower were earlier reported by Khan et al., (2007), Kalukhe et al., (2010), Kumar et al., (2011), Makane et al., (2011), Sudrik et al., (2014) and Rani (2016). For days to 50\% flowering, high heritability coupled high low genetic advance as percent of mean was recorded by 200 ppm followed by 100 ppm of SCG 62 and for days to maturity, $200 \mathrm{ppm}$ of EC 625693 followed by 300 ppm of SCG 62, respectively indicating of non-additive interaction. Arshad (2010) elucidated similar findings for days to $50 \%$ flowering and days to maturity with high heritability and low genetic advance as percent of mean in sunflower.

The traits like plant height, head diameter, seed filling percentage, hull content and 100 grain weight exhibited moderate genotypic and phenotypic coefficient of variation by most of treatments. The above results are in agreement with Rani (2010) in sunflower for plant height, head diameter and seed filling percentage. Whereas, Natikar et al., (2013) observed similar reports for hull content and 100 grain weight in sunflower with moderate variability. High heritability with high genetic advance as percent of mean observed for plant height and head diameter of 100 ppm, 200 ppm and 300 ppm of SCG 62 and results are lined with Kumar et al., (2011), Mahmoud (2012) and Sudrik et al., (2014) in sunflower which creates ample scope for selection. Same treatments exhibits high heritability with moderate genetic advance as percent of mean for hull content and these results are concurred with Kalukhe et al., (2010) in sunflower. The treatments $100 \mathrm{ppm}$ and 300 ppm of EC 625693 showed high heritability and high genetic advance as percent of mean which suggested that improvement of these characters is possible by simple phenotypic selection. These findings confirm the results of Kumar et al., (2011), Mahmoud (2012), Natikar et al., (2013) and Sudrik et al., (2014) in sunflower.

Seed yield per plant showed high genotypic and phenotypic coefficient of variation for most of treatments and high heritability coupled with high genetic advance as percent of mean by 200 ppm and 300 ppm of SCG 62 indicated the presence of additive gene action and it can be improved by simple phenotypic selection. Similar reports in sunflower earlier published by Kumar et al., (2011), Makane et al., (2011), Natikar et al., (2013) and Sudrik et al., (2014).

In general, the mutagenic treatments had an adverse effect on the means of various characters. The study showed that the sodium azide treatments of $100 \mathrm{ppm}$ was found more effective for developing desirable variability in 100 grain weight, seed filling percentage and seed yield per plant, which had high heritability with high genetic advance as percent of mean. Hence, selection for these characters enhances genetic improvement.

\section{References}

Allard, R.W. 1960. Principles of Plant Breeding. John Wiley and Sons. Inc., New York.

Arshad, M., Khan, M.A., Jadoon, S.A. and Mohmand, A.S. 2010. Factor analysis in sunflower (Helianthus annuus L.) to investigate desirable hybrids. Pak. J. Bot., 42(6): 4393-4402.

Burton, G.W. 1952. Quantitative inheritance in grasses. Proc. 6th Inter. Grassland Congre. J., 1; 227-283.

Cvejic, S., Jocic, S., Jockovic, M., Imerovski, I., Dimitrijevic, A., Miladinovic, D. and 
Prodanovic, S. 2015. New genetic variability in sunflower inbred lines created by mutagenesis. Romanian Agricultural Research. 32: 27-34.

Gunasekharan, A. and Pavadai, P. 2015. Studies on induced physical and chemical mutagenesis in groundnut (Arachis hypogaea L.). Inter. Lete. Of Natu. Sciences. 8: 25-35.

Jagadeesan, S., Kandasamy, G., Manivannan, N. and Muralidharan, V. 2008. Mean and variability studies in M1 and M2 generations of sunflower (Helianthus annuus L.) Helia. 31(49): 71-78.

Kalukhe, V.K., Moon, M.K., Magar, N.M. and Patil, S.S. 2010. Genetic variability in sunflower (Helianthus annuus L.). Bioinfolet. 7(3): 197-200.

Khan, H., Muhammad, S., Shah, R. and Iqbal, N. 2007. Genetic analysis of yield and some yield components in sunflower. Sarhad. J. Agric., 23(4): 985-990.

Khan, S. and Goyal, S. 2009. Mutation genetic studies in mungbean IV. Selection of early maturing mutants. Thai J. of Agri. Sci., 42(2): 109-113.

Kulmi, M.R. and Mogali, S.C. 2016. Mutagen induced polygenic variability in linseed (Linus usitatissimum L.). J. Farm. Sci., 29(2): 261-264.

Kumar, G.V., Suresh, J., Kymar, S.S., Saidaiah, P. and Raghu, B. 2011. Genetic variability, heritability and genetic advance for yield and yield components in sunflower (Helianthus annuus L.). J. Oilseeds Res., 28(2): 156158.
Mahmoud, A.M. 2012. Genetic variability in S4 inbred lines of sunflower under sandy soil conditions. AmericanEurasian J. Agric. \& Environ. Sci., 12(3): 282-286.

Makane, V.G., Shinde, C.A., Mohrir, M.N. and Majid, A.B. 2011. Genetic variability studies in new versions of sunflower (Helianthus annuus L.). Bioinfolet. 8(1): 44-51.

Natikar, P., Madhusudan, K., Kage, U., Nadaf, H.I. and Motagi, B.N. 2013. Genetic variability studies in induced mutants of sunflower (Helianthus annuus L.) Plant Gene and Trait. 4(16): 86-89.

Panse, V. G. and Sukhatme, P. V. 1985. Statistical methods for Agricultural Workers, Indian council of Agricultural research, New Delhi. pp- 361.

Rani, M. 2016. Genetic variability and divergence in sunflower (Helinathus annuus L.). M.Sc. (Ag.) Thesis submitted to CCSHAU, Hisar.

Roychowdhury, R., Datta, S., Gupta, P. and Tah, J. 2012. Analysis of genetic parameters on mutant populations of mungbean (Vigna radiate L.) after EMS treatment. Not. Sci. Biol., 4(1): 137-143.

Sudrik, B.P., Ghodke, M.K., Patil, V.S., Chavan, S.K. and Kesale, N.B. 2014. Evaluation and characterization of sunflower (Helianthus annuus L.) germplasm. J. of Crop and Weed. 10(1): 73-76.

\section{How to cite this article:}

Sai Phanindra, K., V.N. Toprope and Thakur, N.R. 2018. Effect of Sodium Azide in Yield and Yield Contributing Traits of Confectionary Sunflower (Helianthus annuus L.). Int.J.Curr.Microbiol.App.Sci. 7(09): 2326-2333. doi: https://doi.org/10.20546/ijcmas.2018.709.289 